\title{
Perfil epidemiológico de cirurgias em serviço de urgência e emergência
}

\author{
Epidemiological profile of surgery in emergency service
}

Carlos Alberto Miranda Lyra ${ }^{1}$ (D), Lara Rebeca Correia Franca Dantas ${ }^{1}$ (D), Stella Costa Todt $^{1}$ (D), Isabella Paiva Palmeira ${ }^{1}$ (D), Maria Bernadete Galrão de Almeida Figueiredo ${ }^{1}$ (D), Sonia Oliveira Lima $^{1}$ (D)

\begin{abstract}
RESUMO
Modelo de estudo: Estudo observacional, analítico e transversal. Objetivo: Analisar o perfil epidemiológico dos pacientes do serviço de urgência e emergência de um hospital privado que foram submetidos a cirurgias. Metodologia: Estudo realizado em um hospital privado com análise de prontuários médicos de pacientes de ambos os sexos com idade igual ou superior a 16 anos que entraram no Serviço de Urgência e Emergência da instituição e foram encaminhados para cirurgia, no período de junho a agosto de 2017. Resultados: Analisou-se 104 prontuários, destes $53,84 \%$ corresponderam ao sexo masculino com média de idade de 46,03 anos. O tempo médio de internação foi 4,22 dias, com desvio padrão 7,01 dias e a mediana de 2 dias. Aqueles que apresentam comorbidades corresponderam a 70,19\%. Houve um óbito de paciente de 83 anos após neurocirurgia com 62 dias de internação $(0,96 \%)$. A maior gama das cirurgias correspondeu à apendicectomia com 25 procedimentos (43,85\%), tempo médio de internação de 2,32 dias e a média de idade de 36,36 anos. Conclusão: Não houve diferença estatística entre os sexos $(p=0.23)$ e a idade média foi de 46 anos com tempo médio de internação de 4 dias. As doenças abdominais e do aparelho locomotor se apresentaram como as principais causas de entrada no serviço de urgência, mantendo o padrão previamente estabelecido na literatura. Das cirurgias por doenças inflamatórias do aparelho digestivo, enfatiza-se a apendicectomia devido à apendicite aguda.
\end{abstract}

Palavras-chave: Epidemiologia; Cirurgia Geral; Serviço Hospitalar de Emergência.

\begin{abstract}
Study model: Observational, analytical, and cross-sectional study. Objective: To analyze the epidemiological profile of patients from the emergency room of a private hospital who underwent surgery. Methodology: Study conducted in a private hospital with analysis of medical records of patients of both sexes aged 16 years or older who entered the institution's Emergency Department and were referred for surgery, from June to August 2017. Results: It was analyzed 104 medical records, of which $53.84 \%$ corresponded to males with a mean age of 46.03 years. The average length of stay was 4.22 days, with standard deviation 7.01 days and median of 2 days. Those with comorbidities corresponded to $70.19 \%$. One patient died 83 years after neurosurgery with 62 days of hospitalization $(0.96 \%)$. The largest range of surgeries corresponded to appendectomy with 25 procedures (43.85\%), mean length of stay of 2.32 days, and mean age of 36.36 years. Conclusion: There was no statistical difference between genders $(p=0.23)$, and the mean age was 46 years with a mean length of stay of 4 days. Abdominal and locomotor disorders were the main causes of admission to the emergency room, maintaining the pattern previously established in the literature. Surgery for inflammatory diseases of the digestive tract emphasizes appendectomy due to acute appendicitis.
\end{abstract}

Keywords: Epidemiology; General Surgery; Emergency Service, Hospital.

1. Universidade Tiradentes (UNIT), Aracaju (SE), Brasil.

$\triangle$ Carlos Alberto Miranda Lyra. Avenida Murilo Dantas, 300 - Farolândia. CEP: 49032-490. Aracaju (SE), Brasil. carlosalbertolyra@hotmail.com | Recebido em: 24/10/2019 | Aprovado em: 21/07/2020 


\section{INTRODUÇÃO}

No Brasil, há grande demanda dos serviços de urgência e emergência, e um dos argumentos utilizados como justificativa para isso é que, parte dos atendimentos realizados decorre de problemas que poderiam ser resolvidos em serviços com menor nível de complexidade, atenção básica, unidades de pronto atendimento ou de ambulatórios especializados. O que se observa, no entanto, é que a grande demanda desses serviços é uma das principais portas de entrada ao sistema de saúde ${ }^{1}$.

Os principais motivos de consulta variam entre os estudos, o que pode ser reflexo das diferenças entre as demandas atendidas e a metodologia aplicada. Rodríguez et al. ${ }^{2}$ observaram que os sinais e sintomas mal definidos e as alterações do aparelho locomotor e conjuntivo foram os motivos mais frequentes de consulta no serviço de emergência. Já Abdallat et al. ${ }^{3}$ identificaram as doenças torácicas e abdominais como principais motivos de consultas em adultos. Esses motivos avaliaram o serviço de emergência hospitalar de uma forma geral, incluindo as áreas clinicas, cirúrgicas, pediátricas e traumatológicas.

Stewart et al. ${ }^{4}$ relataram que 11 condições que requeriam cirurgia de emergência apresentadas pela Global Burden of Disease Study, causaram a morte de 896.000 pessoas no ano de 2010. Dessa maneira, podem-se identificar as causas mais frequentes de internação referentes a pacientes que necessitaram cirurgias de emergência. Isso reforça ideias observadas em outros estudos de que cirurgias de urgências são componentes importantes no modelo de cuidados de um paciente 5 .

Então, ao se analisar a abrangência que o setor de urgência e emergência alcança, tem-se uma perspectiva da importância deste para o sistema de saúde de um país. Assim sendo, uma das melhores formas de entender as condições de saúde que levam a população a uma urgência, seja ela geral ou específica como a cirúrgica, é através de dados epidemiológicos, onde se pode identificar as causas mais frequentes e observar os padrões vistos $^{6}$

Estudos com o objetivo de analisar dados epidemiológicos, geralmente utilizam-se de metodologias de análise de prontuários de um determinado período com variáveis como queixa, idade, sexo, comorbidades, dentre outras, que vão refletir a atual demanda daquele hospital estudado, sendo capaz, assim, de avaliar a causa e resultado daquela hospitalização. A maioria dessas pesquisas trata de doenças específicas, e não aos atendimentos e cirurgias de urgência e emergência em uma maneira geral ${ }^{1,2,4}$. Em vista da relevância deste setor, do destaque que o componente cirúrgico exerce no cuidado agudo e do fato de existirem dados limitados que definem a epidemiologia dos pacientes do serviço de urgência e emergência, há uma necessidade de entender a demanda desse campo. Objetivou-se, portanto, analisar o perfil epidemiológico dos pacientes do serviço de urgência e emergência de um hospital privado que foram submetidos a cirurgias, relacionar os tipos de cirurgias realizadas com o tempo de internação, associar a mortalidade com o tipo de cirurgia e correlacionar a mortalidade com a causa de morte.

\section{MÉTODOS}

Trata-se de um estudo observacional, analítico e transversal realizado em um hospital privado e acreditado da cidade de Aracaju-SE. Após aprovação pelo Comitê de Ética em Pesquisa sob no 2.252.677, foram analisados prontuários médicos de pacientes que entraram no Serviço de Urgência e Emergência da instituição e foram encaminhados para cirurgia, no período de junho a agosto de $2017(n=131)$. Destes foram selecionados apenas os pacientes com idade igual ou maior a 16 anos $(n=104)$.

As variáveis analisadas e obtidas por meio do prontuário do paciente, foram: número do prontuário, sexo, data de nascimento, CID-10 no momento da internação, tipo de cirurgia realizada, tempo de internação, mortalidade, causa da mortalidade e morbidades associadas.

Inicialmente, os pacientes foram separados em grupos (Tabela 2 ) de acordo com CID-10, no momento de internação. Foram calculadas as médias, comparando-as através do teste não paramétrico de Wilcoxon-MannWhitney. Considerou-se como nível de significância $p<0,05$, sendo empregado o software estatístico SPSS versão 22.0 (SPSS, Chicago, EUA) para análise dos dados. 


\section{RESULTADOS}

A análise do perfil epidemiológico demonstrou que $53,84 \%$ (56) dos pacientes foi do sexo masculino e a média de idade de 46,03 anos. 0 tempo médio de internação foi de 4,22 dias, já o desvio padrão foi de 7,01 dias e a mediana foi de 2 dias (Tabela 1). Foi então utilizado um teste não paramétrico de Wilcoxon-Mann-Whitney para comparação de médias do tempo de internamento do grupo de pacientes do sexo masculino em relação ao de pacientes do sexo feminino ( $W=1173.5$, $p=0.23$ ). Não foi encontrada diferença estatisticamente significativa entre as médias dos grupos testados, desfavorecendo um possível modelo de dependência da variável de tempo de pós-operatório em função do sexo do paciente.

\section{Tabela 1}

No de Dias de Internação das Cirurgias de Urgências de um Hospital Privado em Aracaju-SE entre Junho e Agosto de 2017

\begin{tabular}{lc}
\hline Valor Máximo & 62 \\
Valor Mínimo & 1 \\
Média & 4,221153846 \\
Desvio Padrão & 7,01725575 \\
Mediana & 2 \\
\hline
\end{tabular}

Fonte: Dados próprios

Aqueles que apresentam comorbidades corresponderam a 70,19\% (73). Uma paciente de 96 anos, após neurocirurgia e com 62 dias de internação foi a óbito devido a uma parada cardiorrespiratória $(0,96 \%)(1)$.

A maior gama das cirurgias correspondeu a apendicectomias, devido a apendicites agudas, que equivaleram a 25 procedimentos $(43,85 \%)$. A média de tempo de internação destes pacientes foi de 2,32 dias, apresentando mediana de 2 dias, valor máximo de 5 dias e valor mínimo de 1 dia. Quanto a média de idade, esta foi de 36,36 anos.

As cirurgias ortopédicas representaram o segundo maior percentual, com 18 procedimentos $(17,30 \%)$. Deste total, $5(27,77 \%)$ são devido a fratura da perna, incluindo o tornozelo. A média de idade destes pacientes foi maior do que aqueles submetidos a apendicectomias, demonstrando um total de 47,83 anos, e a média do tempo de internação seguiu o mesmo entendimento, com 3,72 dias.

As doenças das vias biliares representaram a terceira maior causa de entrada na emergência, com 12 casos $(11,53 \%)$, e outras afecções do aparelho digestivo foram responsáveis por 11 procedimentos $(10,57 \%)$. O restante dos tratamentos cirúrgicos foi devido a neoplasias $(1,92 \%)(2)$, hérnias $(4,80 \%)(5)$, sepse $(3,84 \%)(4)$, dor abdominal inespecífica $(8,65 \%)(9)$, doenças do aparelho reprodutor feminino (2,88\%) (3), doenças do aparelho reprodutor masculino $(0,96 \%)(1)$, hemorragias intracranianas $(3,84 \%)(4)$, doenças crônicas $(0,96 \%)(1)$, doenças torácicas $(2,88 \%)(3)$, doenças vasculares periféricas $(1,92 \%)(2)$ e doenças do aparelho urinário (3,84\%) (4) (Tabela 2).

\section{Tabela 2}

Causas de Internação para Cirurgias de Urgência entre Junho e Agosto de 2017 de um Hospital Privado em Aracaju-SE

\begin{tabular}{lcc}
\hline Causa & N & Frequência (\%) \\
\hline Neoplasias & 2 & $1,92 \%$ \\
Apendicite Aguda & 25 & $24,04 \%$ \\
Vias Biliares & 12 & $11,54 \%$ \\
Hérnias & 5 & $4,81 \%$ \\
Outras doenças do aparelho & 11 & $10,58 \%$ \\
digestivo & 4 & $3,85 \%$ \\
Sepse & 9 & $8,65 \%$ \\
Dor abdominal inespecífica & 3 & $2,88 \%$ \\
Aparelho reprodutor feminino & 3 & $3,85 \%$ \\
Hemorragias intracranianas & 4 & $0,96 \%$ \\
Doenças crônicas & 1 & $2,88 \%$ \\
Doenças torácicas & 3 & $17,31 \%$ \\
Fraturas & 18 & $1,92 \%$ \\
Doenças vasculares & 2 & $3,85 \%$ \\
periféricas & & $0,96 \%$ \\
Aparelho urinário & 4 & $100,00 \%$ \\
Aparelho reprodutor & 1 & \\
masculino & & \\
Total & 104 &
\end{tabular}

Fonte: Dados próprios

\section{DISCUSSÃO}

A apendicectomia e a colecistectomia foram responsáveis pela maior parte dos procedimentos 
realizados, correspondendo a 55,38\% (58) do total de cirurgias. Semelhante a Scott et al. 7 que verificaram que as cirurgias mais realizadas, no departamento de emergência nos EUA foram colectomia parcial, ressecção do intestino delgado, colecistectomia, tratamento cirúrgico da úlcera péptica, lise das aderências peritoneais, apendicectomia e laparotomia. Estes representaram $80,0 \%$ de todos os procedimentos, $80,3 \%$ de todas as mortes, $78,9 \%$ de todas as complicações e $80,2 \%$ de todos os custos de pacientes nesse país. De acordo com Snyder et al. ${ }^{8}$, os motivos mais comuns para as cirurgias de emergência num hospital moçambicano foram laparotomias não traumáticas (22\%), trauma (18\%), apendicectomia (15\%), correção de hérnia $(13 \%)$, amputação (12\%) e incisão e drenagem (11\%).

A apendicectomia correspondeu a $43,85 \%$ $(n=25)$ dos procedimentos realizados, tendo uma média de idade de 36,36 anos e média de tempo de internação de 2,32 dias, não apresentando diferença estatística na prevalência dos sexos dos pacientes. Contrastando com estudos como os de Ceseroli et al. ${ }^{9}$, que encontrou uma média de idade de 25,04 anos e prevalência maior no sexo masculino e de Lee et $a{ }^{10}$.que observou maior número de casos de pacientes sul-coreanos entre 10 e 19 anos, mantendo a prevalência do sexo masculino. No entanto, também se verifica que há semelhanças encontradas em outras regiões do Brasil e dados americanos, os quais mostraram uma média de idade em torno de 35 anos e uma leve incidência maior nos pacientes do sexo masculino ${ }^{11,12}$. Coelho et al. ${ }^{11}$ ainda verificaram semelhança no tempo médio de internação dos pacientes submetidos a esse procedimento: em hospitais públicos brasileiros foi de $3,5 \pm 2,8$ dias, enquanto em hospitais privados foi de 2,5 \pm $1,7$ dias ( $p=0,0024)$.

As cirurgias ortopédicas corresponderam a $17,30 \%$ (18) dos procedimentos, sendo $27,77 \%(5)$ devido a fratura da perna, incluindo o tornozelo. Semelhante a este estudo, Donaldson et al. ${ }^{13}$ observaram que as fraturas mais comuns são dos pés e mãos, seguidas por fraturas de ossos longos e tronco. Esta assertiva não foi encontrada em outro estudo, como o de Boufous et al. ${ }^{14}$ que aponta fraturas de quadril $(86,5-96,1 \%)$, pélvicas $(67,8-82,6 \%)$ e punho $(26,2-28,9 \%)$ como as mais comuns. Outra diferença a respeito das cirurgias ortopédicas pode ser notada quando se avalia a média de idade encontrada na literatura. Estudos como o de Donaldson et al. ${ }^{13}$ e Boufous et al. ${ }^{14}$., demonstraram que a incidência de fraturas apresenta picos diferentes entre os sexos, mas tem em comum um aumento com a idade, principalmente em pessoas acima de 80 anos.

A baixa mortalidade encontrada no presente estudo pode ser reflexo da instituição do estudo ser acreditada, possuir profissionais qualificados e não ser referência em trauma no estado, com baixa demanda de pacientes politraumatizados.

\section{CONCLUSÃO}

Não houve diferença estatística entre os sexos $(p=0.23)$ e idade média de 46 anos com tempo médio de internação de 4 dias. As doenças abdominais e do aparelho locomotor se apresentaram como as principais causas de entrada no serviço de urgência, mantendo o padrão previamente estabelecido na literatura. Das cirurgias por doenças inflamatórias do aparelho digestivo, enfatiza-se a apendicectomia devido à apendicite aguda. Isto demonstra que a epidemiologia previamente estabelecida manteve seu padrão, corroborando com os dados da literatura.

\section{REFERÊNCIAS}

1. Simons DA. Avaliação do perfil da demanda na unidade de emergência em alagoas a partir da municipalização da saúde e do programa saúde da família. Recife. Tese (Doutorado em Saúde Pública). Fundação Oswaldo Cruz, Centro de Pesquisa Aggeu Magalhães; 2008.

2. Rodríguez JP, Sánchez ID, Rodríguez RP, Acosta AS. "Filtro sanitario" en las urgencias médicas: Un problema a reajustar. Rev cubana med 2001 Sep; 40(3):181-188.

3. Abdallat AM, et al. Who uses the emergency room services? Eastern Mediterranean Health Journal. 2000; 6(5-6): 1126-1129.

4. Stewart B, Khanduri P, McCord C, Ohene-Yeboah M, Uranues $S$, Vega Rivera $F$, et al. Global disease burden of conditions requiring emergency surgery: Global disease burden of conditions requiring emergency surgery. British Journal of Surgery. 2014 Jan;101(1):e9-22.

5. Narayan M, Tesoriero R, Bruns BR, Klyushnenkova EN, Chen H, Diaz JJ. Acute Care Surgery: Defining Mortality in Emergency General Surgery in the State of Maryland. 
Journal of the American College of Surgeons. 2015 Apr;220(4):762-70.

6. Freire $A B$, Fernandes DL, Moro JS, Kneipp MM, Cardoso CM, Lima SBS. Serviços de urgência e emergência: quais os motivos que levam o usuário aos pronto-atendimentos? Saúde (Santa Maria) Jun 2015; 41(1):195-200.

7. Scott JW, Olufajo OA, Brat GA, Rose JA, Zogg CK, Haider $A H$, et al. Use of National Burden to Define Operative Emergency General Surgery. JAMA Surgery. 2016 Jun 15;151(6):e160480.

8. Snyder E, Amado V, Jacobe M, Sacks GD, Bruzoni M, Mapasse D, et al. General surgical services at an urban teaching hospital in Mozambique. Journal of Surgical Research. 2015 Oct;198(2):340-5.

9. Ceresoli M, Zucchi A, Allievi N, Harbi A, Pisano M, Montori G, et al. Acute appendicitis: Epidemiology, treatment and outcomes- analysis of 16544 consecutive cases. World Journal of Gastrointestinal Surgery. 2016;8(10):693.
10. Lee JH, Park YS, Choi JS. The Epidemiology of Appendicitis and Appendectomy in South Korea: National Registry Data. Journal of Epidemiology. 2010;20(2):97-105.

11. Coelho JCU, Fernandes FM, Cortiano LGG, Leme GM de $\mathrm{O}$, Sadowski JA, Artner CL. Appendectomy: comparative study between a public and a private hospital. Revista da Associação Médica Brasileira. 2010;56(5):522-7.

12. Metcalfe D, Olufajo O, Rios-Diaz AJ, Haider A, Havens JM, Nitzschke $S$, et al. Are appendectomy outcomes in level I trauma centers as good as we think? Journal of Surgical Research. 2016 May;202(2):239-45.

13. Donaldson LJ, Reckless IP, Scholes S, Mindell JS, Shelton NJ. The epidemiology of fractures in England. Journal of Epidemiology \& Community Health. 2008 Feb $1 ; 62(2): 174-80$.

14. Boufous S, Finch C, Close J, Day L, Lord S. Hospital admissions following presentations to emergency departments for a fracture in older people. Injury Prevention. 2007 Jun 1;13(3):211-4. 